\title{
Determinación de Babesia canis y Ehrlichia canis, en hemolinfa de garrapatas teleoginas de Riphicephalus sanguineus
}

\author{
Determination of Babesia canis and Ehrlichia canis, in hemolymph of \\ teleogynous ticks of Riphicephalus sanguineus \\ Gonzales Barbosa Liliana ${ }^{1}$ y Lesmes Luis Alfonso ${ }^{1}$ \\ ${ }^{1}$ Medico Veterinario Zootecnista, Universidad de los Llanos \\ llesmes@laboratoriosprovet.com.co
}

Recibido 05 de Marzo 2013, Aceptado 02 de Abril 2013

\section{RESUMEN}

El presente estudio se llevó a cabo en el laboratorio de microbiología de la Universidad de los Llanos Orientales, ubicada a $4 \mathrm{~km}$ de la ciudad de Villavicencio (Meta), con una temperatura promedio de $28^{\circ} \mathrm{C}$, humedad relativa del $80 \%$ y una altura de $440 \mathrm{msnm}$. El objetivo fue determinar la presencia de Babesia canis y Ehrlichia canis, por medio del extendido y coloración de Giemsa en la hemolinfa de garrapatas teleoginas de Riphicephalus sanguineus (RS), mediante capturadas en perros de la ciudad de Villavicencio, utilizando la técnica de identificación propuesta por López (1980); con el fin de conocer si la técnica es aplicable para esta garrapata. Para el estudio se recolectaron 200 garrapatas teleoginas de RS, las cuales se tomaron de caninos infestados, en la ciudad de Villavicencio. Para el procedimiento del examen de hemolinfa se lavaron las garrapatas con agua y alcohol antiséptico al $70 \%$, luego fueron secadas con toalla de papel introduciéndolas en cajas de petri para colocarlas a incubar a $28^{\circ} \mathrm{C}$ y $80 \%$ de humedad relativa por tres días. Se procedió a cortar los tarsos cuidadosamente de cada una de las garrapatas en incubación, extrayendo de estos una muestra de hemolinfa. Esta gota de hemolinfa se colocó en láminas porta objetos, se procedió a fijarla con alcohol metílico por 2 minutos, luego se inició la coloración con Giemsa por 45 minutos, posteriormente se observaron al microscopio (100x) y se identificaron las células de la hemolinfa. Las muestras analizadas de hemolinfa de RS, arrojaron un resultado del $100 \%$ negativas para los dos agentes patógenos en 
estudio Babesia canis y Ehrlichia canis. Aunque no se determinó la presencia de Ehrlichia canis y Babesia canis en los extendidos de hemolinfa tomados del tarso de las teleoginas de RS en los muestreos realizados no se puede asumir que los hemoparásitos no se encuentran presentes en los caninos, por esta razón es recomendable realizar muestreos de las garrapatas en diferentes fases del ciclo de vida, con base en estudios realizados en la hemolinfa de RS variando la temperatura. Se concluye que la técnica descrita por López en 1980, no es aplicable para la determinación de Babesia canis y Ehrlicha canis en las condiciones desarrolladas en el presente trabajo.

Palabras clave: Hemoparásitos, Babesia canis, Ehrlichia canis, Riphicephalus sanguineus.

\section{ABSTRACT}

The present study was carried out in the laboratory of microbiology at the University of the Llanos Orientales, located $4 \mathrm{~km}$ from the city of Villavicencio (Meta), with an average temperature of $28^{\circ} \mathrm{C}$, relative humidity of $80 \%$ and a height of 440 m.a.s.l. The objective was to determine the presence of Babesia canis and Ehrlichia canis, through extended and Giemsa in the hemolymph of engorged females of Rhipicephalus sanguineus ticks (RS) by dogs captured in the city of Villavicencio, using the proposed identification technique by Lopez (1980) in order to determine whether the technique is applicable to this tick. For the study collected 200 ticks engorged RS, which were taken from infected canines in the city of Villavicencio. For hemolymph-examination ticks were washed with water and $70 \%$ rubbing alcohol, then were dried with paper towel petri dishes fitting them in to place to incubate at $28^{\circ} \mathrm{C}$ and $80 \%$ relative humidity for three days. He proceeded to cut the shanks carefully each incubation ticks, extracting from them a sample of hemolymph. This drop of hemolymph was placed on slide slides, fixed with methyl alcohol for 2 minutes, then began staining with Giemsa for 45 minutes, then observed under a microscope (100x) and cells were identified by the hemolymph. The samples of hemolymph RS, yielded a negative result of $100 \%$ for both study pathogens Babesia canis and Ehrlichia canis. Although not determined the 
presence of Ehrlichia canis and Babesia canis in hemolymph smears taken from the tarsus of the RS engorged in the samples taken cannot be assumed that blood parasites are not present in canines, for this reason it is advisable to perform sampling of ticks in different phases of the life cycle, based on studies in the hemolymph of RS varying the temperature. We conclude that the technique described by Lopez in 1980, does not apply to the determination of Babesia canis and Ehrlicha canis in the conditions developed in the present work.

Keywords: Hemoparasite, Babesia canis, Ehrlichia canis, Riphicephalus sanguineus.

\section{INTRODUCCIÓN}

En su labor, los Médicos Veterinarios se ven enfrentados a enfermedades bacterianas, virales, parasitarias, entre otras; muchas de éstas transmitidas por diferentes vectores, los cuales pueden servir como hospedadores intermediarios importantes, que permiten la prevalencia de la enfermedad y el incremento de las enfermedades zoonóticas. El laboratorio clínico, es una de las herramientas más utilizadas para el diagnóstico de las enfermedades hemoparasitarias, ya que ayuda al clínico a enfocar el diagnostico, sin embargo, la identificación del agente etiológico de la enfermedad hemoparasitaria, junto con el análisis de los signos clínicos y los resultados de patología clínica, permite el diagnóstico definitivo de la enfermedad, situación que se logra en pocos casos.

La Babesiosis y la Ehrlichiosis, son dos enfermedades hemoparasitarias frecuentes en perros en los llanos orientales, transmitidas principalmente por Riphicephalus sanguineus (RS) (López, 1999), con signos clínicos comunes con otras enfermedades, que hacen en la praxis que se incluyan varios diagnósticos diferenciales, esto obliga al Médico Veterinario a apoyarse en el laboratorio clínico, con el fin de orientar su diagnóstico.

La Babesiosis es una enfermedad infecciosa causada por un protozoo llamado Babesia intraeritrocítico (Cholich et al., 2004). La babesiosis en el perro es causada por Babesia canis. Estudios recientes han demostrado que existen tres 
genotipos en todo el mundo, Babesia canis, Babesia canis vogeli y Babesia canis rossi, de las cuales las tres pueden ser transmitidas por RS. La Babesia canis se observa como trofozoítos piriformes en glóbulos rojos, siendo observados también en macrófagos y células endoteliales de pulmón e hígado (Hendrix, 1999). El cuadro clínico varía desde cuadros fulminantes a leves, incluso autolimitante, por lo que la enfermedad suele pasar desapercibida y, en muchas ocasiones, ni siquiera se diagnostica (Couto y Nelson, 2000). El diagnóstico se realiza mediante un análisis de sangre que permite identificar a los parásitos en el interior de los glóbulos rojos: Frotis sanguíneo teñida con Giemsa. Si no se observa no se puede descartar y entonces la serología es la base del diagnóstico. El tratamiento de la enfermedad se escoge en función del cuadro clínico. Si el cuadro es severo hay que actuar con urgencia y establecer fluidoterapia intravenosa e incluso transfusión en caso de anemia severa. Si el animal está estable se utiliza dipropionato de imidocarb con una dosis de $5 \mathrm{mg} / \mathrm{Kg}$ de peso vía intramuscular con previa aplicación de Atropina y se repite la dosis a los 15 días. La forma de prevención principal de esta enfermedad es el tratamiento contra las garrapatas (Aguirre et al., 2004)

La Ehrlichiosis es otra enfermedad infecciosa causada por un microorganismo parásito obligado intracelular perteneciente a la familia Rickttsiaceae denominado Ehrlichia canis. Este es un microorganismo pleomórfico, cocoide gram (-), aeróbico que no crece en medios bacteriológicos estándares. Se caracteriza por la sobrevivencia intracelular obligada localiza dentro del citoplasma y se reproduce por fisión binaria tanto en el hospedero vertebrado como en el vector invertebrado (Simone et al., 2005). Existen diferentes especies dentro de este grupo siendo Ehrlichia canis la principal responsable de los cuadros clínicos en el perro. Estos agentes son transmitidos por la picadura de garrapatas y en casos esporádicos, por transfusión de sangre (Dalgliesh et al., 1998). Básicamente existen tres presentaciones clínicas de Ehrlichiosis canina (EC). Estas se diferencian porque las especies causantes tienen afinidad variable por distintos tipos de células sanguíneas (Couto y Nelson, 2000). Las principales son E. monocítica: afecta a células sanguíneas mononucleares produciendo un descenso en el número de 
todas ellas. Las especies productoras de este proceso (entre ellas E. canis) son las responsables de los cuadros clínicos más importantes y típicos de la enfermedad. E. granulocítica: afecta principalmente a los neutrófilos y los signos clínicos son cojera, inflamación de articulaciones y fiebre. Trombocitopenia cíclica: sin signos clínicos evidentes, únicamente se encuentra descenso en el número de plaquetas. La EC es transmitida por RS o garrapata café del perro por lo que la enfermedad podría presentarse donde quiera que este parásito se encuentre, principalmente donde el clima es tropical (Barrera et al., 2005). Se ha determinado que en zonas endémicas la seroprevalencia no difiere significativamente entre perros mascotas clínicamente sanos con perros callejeros o que presenten sintomatología clínica (Woody y Hoskins, 1991). La transmisión se realiza mediante un vector biológico y por lo tanto la Ehrlichiosis puede ocurrir donde quiera que se encuentre el vector. Una vez infectada la garrapata puede transmitir la enfermedad por lo menos 155 días después que se separa del hospedero, es decir, que puede ocurrir en cualquier estado de desarrollo de la garrapata (larva, ninfa, adulto). La diseminación entre ellas no es transovárica sino a través de un perro infectado. La infección en el canino se desarrolla cuando la garrapata se alimenta de la sangre y su secreción salival contamina el sitio donde pica (Cordovés, 1997). La gravedad y presentación de los signos clínicos dependen del propio sistema inmunitario de cada animal y de la virulencia del agente infeccioso, éste se multiplica en células mononucleares circulantes y en células de defensa del hígado y bazo (sistema fagocítico mononuclear). De estas células puede transportarse a otros órganos como pulmones, meninges, riñones. Del tipo de lesión y localización en los diferentes órganos y sistemas derivan los signos clínicos que manifiesta el animal infectado (Hendrix, 1999). El diagnóstico se realiza en una primera etapa, con base en la sintomatología clínica y al cuadro hematológico, donde encontramos pancitopenia, anemia aplásica y trombocitopenia; ésta última es considerada como la alteración más consistente en la infección por Ehrlichia canis. El examen serológico mediante Inmunofluorescencia Indirecta (IFAT) constituye el método de elección para el diagnóstico de EC. Por último, está el diagnóstico citológico que se realiza por la 
observación de la mórula de Ehrlichia canis en los monocitos. Los exámenes hematológicos no siempre entregan una información clara, suele observarse leucopenia seguida por leucocitosis con monocitosis y linfocitosis. Generalmente la anemia es no regenerativa. Estas anormalidades hematológicas rara vez se presentan en forma simultánea, son más frecuentes diversas combinaciones (de Tami y Tami, 2004). El tratamiento consiste en la administración de tetraciclina u oxitetraciclina y en el caso de infecciones crónicas con evidencia de falla renal, la doxiciclina (Hoskins, 1991). Oxitetraciclina por vía oral en dosis de $33 \mathrm{mg} / \mathrm{kg}$. TID durante 2 - 4 semanas, dependiendo del caso en particular, más administración de tratamiento de sostén (transfusión sanguínea, electrolitos, vitaminas) de acuerdo a

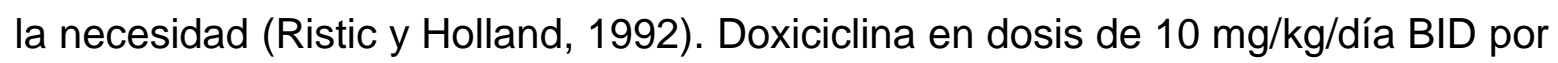
un mes en casos agudos y en casos crónicos por dos meses (Greene, 1995).

Este trabajo busca determinar la presencia de Babesia canis y Ehrlichia canis, por medio del extendido y coloración de Giemsa en la hemolinfa de garrapatas teleoginas de RS, capturadas en perros de la ciudad de Villavicencio, utilizando la técnica de identificación propuesta por López (1980); con el fin de conocer si la técnica es aplicable para esta garrapata.

Evaluar y desarrollar técnicas de identificación de los agentes hemoparasitarios en su hospedador, permite el desarrollo de investigación básica, con el fin que más adelante sea correlacionada la evidencia de los hospedadores contaminados, con los animales positivos a la enfermedad hemoparasitaria, lo que puede convertir la evaluación de la garrapata, en una prueba diagnóstica indirecta y económica en el caso que la parasitemia por Babesia canis y Ehrlichia canis en el perro sea baja y no permita su identificación en los extendidos de sangre periférica.

\section{METODOLOGÍA}

El presente estudio se llevó a cabo en el laboratorio de microbiología de la Universidad de los Llanos Orientales, ubicada a $4 \mathrm{~km}$ de la ciudad de Villavicencio (Meta), con una temperatura promedio de $28^{\circ} \mathrm{C}$, humedad relativa del $80 \%$ y una altura de 440 msnm (IDEAM, 2003). Para el estudio se recolectaron 200 
garrapatas Teleoginas de RS, las cuales se tomaron de caninos infestados (Figura 1), en la ciudad de Villavicencio. Las Teleoginas adultas de RS que se colocaron en una caja de petri para luego llevarlas a la cámara húmeda y se dejaron en incubación a $28^{\circ} \mathrm{C}$ y un $80 \%$ de humedad relativa por cinco días para Babesia canis.

Para el procedimiento del examen de hemolinfa en la garrapata RS se continuó con el siguiente protocolo: se lavaron las garrapatas con agua y alcohol antiséptico al $70 \%$ (Figura 2), luego fueron secadas con toalla de papel introduciéndolas en cajas de petri para colocarlas a incubar a $28^{\circ} \mathrm{C}$ y $80 \%$ de humedad relativa por tres días ya que a los cinco días lo invertebrados se encontraban ovopositados.

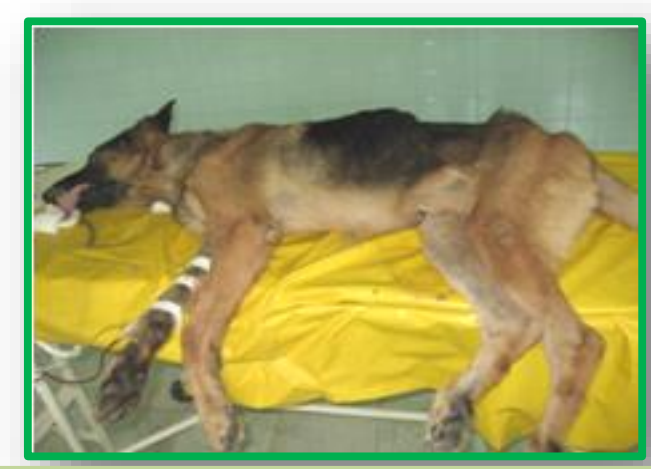

Figura 1. Animal para extracción de las garrapatas

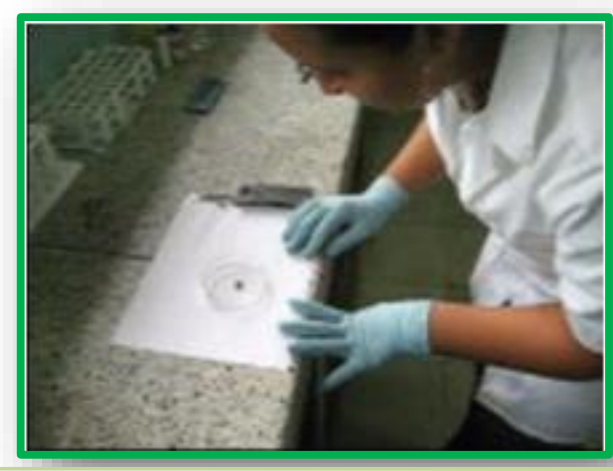

Figura 2. Lavado de la garrapata con agua y alcohol antiséptico al $70 \%$

Se procedió a cortar los tarsos cuidadosamente de cada una de las garrapatas en incubación (Figura 3), extrayendo de estos una muestra de hemolinfa. Esta gota de hemolinfa se coloca en láminas porta objetos, previo diseño del círculo con esmalte en la parte anterior de la laminilla (Figura 4), para tener un mejor reconocimiento dado la escasez de la muestra.

Cuando se tuvo la muestra de hemolinfa dentro del círculo, se procedió a fijarla con alcohol metílico por 2 minutos (Figura 5). Luego se inició la coloración con Giemsa por 45 minutos (Figura 6), tiempo para terminar la fijación, cuando las láminas secaron completamente se observó al microscopio (100x) y se identificaron las células de la hemolinfa. 


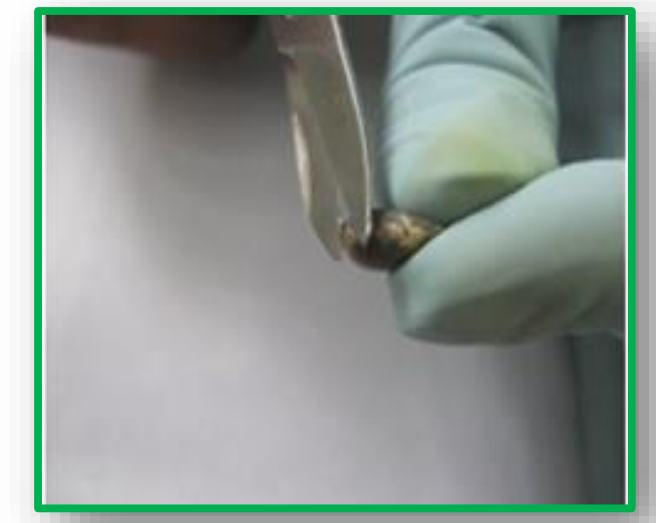

Figura 3. Corte con tijeras para obtención de los tarsos

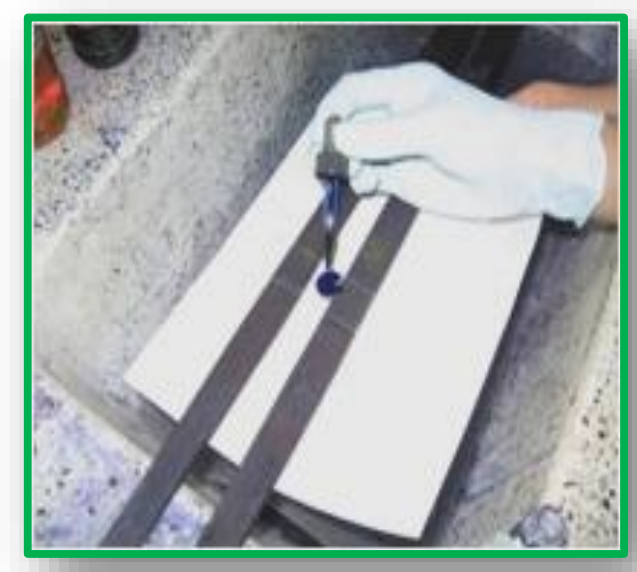

Figura 5. Fijación de la Hemolinfa con alcohol metílico

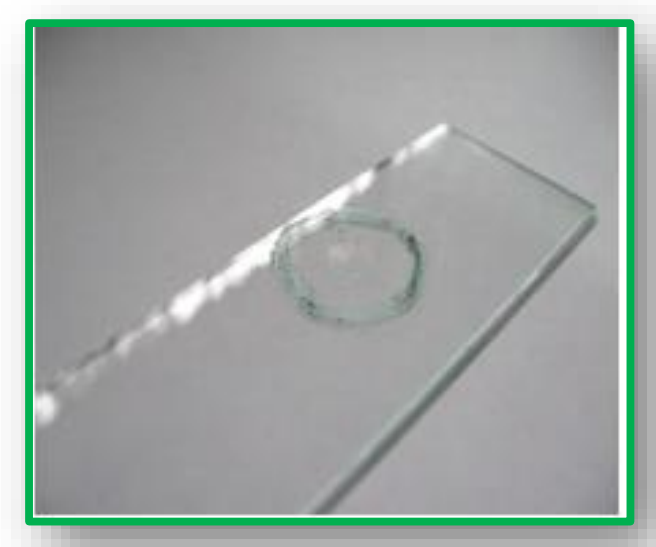

Figura 4. Demarcación lamina para muestra de Hemolinfa

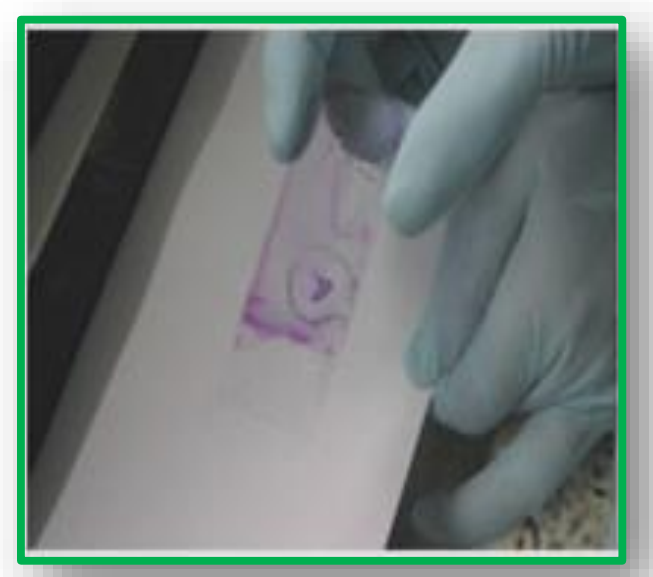

Figura 6. Coloración Giemsa

\section{RESULTADOS Y DISCUSIÓN}

Dentro del desarrollo de la toma de muestras y ejecución del proyecto se tomaron 200 garrapatas a las cuales se les realizó el corte de sus tarsos para la extracción de hemolinfa, representadas en animales infestados con la enfermedad y aparentemente sanos. Las muestras analizadas de hemolinfa de RS, arrojaron un resultado del $100 \%$ negativas para los dos agentes patógenos en estudio Babesia canis y Ehrlichia canis. Se redujo el tiempo de incubación de 5 días a 3 días ya que las garrapatas se encontraban ovopositadas en el primer lapso de tiempo. 
Babesia canis no se determinó en los extendidos de hemolinfa tomados de la garrapata $R S$, la no evidencia de este hemoparásito puede indicar que la garrapata en ninguno de sus estados del ciclo de vida se infectó con los kinetos los cuales van al lumen del intestino y de allí de una forma no establecida van hacia las glándulas salivales donde se trasforman en esporozoitos infectivos, de igual manera se desplazan a todas las estructuras de la garrapata incluyendo la hemolinfa.

Ehrlichia canis no se determinó en los extendidos de hemolinfa tomados de la garrapata RS, lo que puede indicar que estas garrapatas en ninguna fase de su ciclo fueron infectadas con este hemoparásito, puesto que de lo contrario se evidenciaría el hemoparásito en el extendido de hemolinfa, ya que la transmisión de Ehrlichia canis en la garrapata es de tipo trans-estadial, es decir, de larva a ninfa y de ninfa a adulto, sin que se haya podido demostrar hasta el momento la existencia de transmisión transovárica (Woody y Hoskins, 1991).

La no evidencia tanto de Babesia canis como de Ehrlichia canis también puede estar ligada a las condiciones medioambientales ya que se sabe que afectan el ciclo de la garrapata directamente, tal como lo evidencio Benavides, (1982) coincidiendo con lo encontrado por López, (1980) por este motivo se plantea la posibilidad de que también altere el ciclo biológico de los protozoos.

Con respecto a la técnica descrita por López, (1980) la cual consiste en determinar la presencia de los hemoparásitos por medio del extendido de hemolinfa procedente del corte del tarso de la garrapata, previo lavado e incubación por 5 días, no se pudo llevar a cabalidad esta técnica puesto que las garrapatas recolectadas, después del lavado se colocaban a incubar por los cinco días que se describen pero se observó que éstas comenzaban ovoposición entre los 2 a 3 días con lo cual, las garrapatas al día 5 llegaban con alto grado de deshidratación y un alto porcentaje de ovoposición y la muestra obtenida de hemolinfa no fue suficiente para hacer el extendido en la mayoría de los casos, esto se le atribuyó a las condiciones que se dieron a las garrapatas como humedad y temperatura constantes, favoreciendo que su desarrollo de ovoposición fuese más rápido; esto 
contradice lo mencionado por (Barreira, 2005) el cual afirma que entre el $5^{\circ}$ y el $17^{\circ}$ día de incubación son los indicados para la obtención de muestras de hemolinfa. Por tal razón se decidió hacer la modificación de la técnica descrita por López en el tiempo de incubación y tomar las muestras al tercer día puesto que las garrapatas al quinto día se encontraban ovopositadas.

En animales posiblemente infectados y asintomáticos, no se realizó diagnostico confirmativo de los mismos con respecto a babesiosis y ehrlichiosis, lo que obliga a realizar el mismo estudio en garrapatas procedentes de animales con diagnóstico de babesiosis y ehrlichiosis confirmados y con garrapatas en diferentes estados de desarrollo que permita aclarar si la técnica aplica para RS.

\section{CONCLUSIONES}

Aunque no se determinó la presencia de Ehrlichia canis y Babesia canis en los extendidos de hemolinfa tomados del tarso de las teleoginas de Riphicephalus sanguineus en los muestreos realizados no se puede asumir que los hemoparásitos no se encuentran presentes en los caninos, por esta razón es recomendable realizar muestreos de las garrapatas en diferentes fases del ciclo de vida, basados en estudios realizados en la hemolinfa de Riphicephalus sanguineus variando la temperatura y observando cambios en la línea de células encontradas en la fase de larva, tal como lo reportan Carneiro y Daemon en 2003.

La no evidencia de los hemoparásitos pudo estar ligada a que los perros de donde se tomaron las garrapatas estaban aparentemente sanos y no presentaban sintomatología compatible con las entidades, también a que la técnica descrita en la metodología no se realizó en su totalidad pues la incubación de las garrapatas solo duraba entre dos y tres días antes de que se muestrearán las garrapatas.

La técnica descrita por López en 1980, no es aplicable para la determinación de Babesia canis y Ehrlicha canis en las condiciones desarrolladas en el presente trabajo. 


\section{REFERENCIAS BIBLIOGRÁFICAS}

1. Aguirre, D. H., Cafrune M. M., Rada, M., Torioni de E. S. Babesiosis clínica en caninos. Cerillos, Argentina. p. 124-125. 2004.

2. Almeira K. Garrapatas un peligro para la salud animal. En: www.scielo.com Instituto Butantan, São Paulo, Brasil. 2003.

3. Baker A. S. Mites and ticks of domestic animals. An identification guide and information source. $1^{\text {st }}$ ed. The Natural History Museum. The Stationary office. London. 240 p. 1999.

4. Barreira J., Rossi M. I., Pires, F. A. et al. Dynamic of infection of Babesia bovis in engorged females and eggs of Boophilus microplus. Cienc. Rural, 35 (5): 1131-1135. 2005.

5. Barriga O. O. Veterinary Parasitology. The Ohio State University. 297 p. 1994.

6. Benavides O. E. Biología ovoposicional de la garrapata Boophilus microplus en condiciones de los Llanos orientales de Colombia. En: Revista ICA. 1984.

7. Breitschwerdt E. B., Hegarty B. C., Hancock S. I. Sequential evaluation of dogs naturally infected with Ehrlichia canis, Ehrlichia chaffeensis, Ehrlichia equi, Ehrlichia ewingii, or Bartonella vinsonii, J. Clin. Microbiol, 36: 2645-2651. 1998.

8. Castillo A., López J., Hallazgos de Ehrlichia canis en Chile, Valdivia, Chile volumen 31 n.2. 1999.

9. Codner E. C., Farris S. L. L. Characterization of the subclinical phase of ehrlichiosis on dogs, Am. J. Vet. Res., 50: 1544. 1989.

10. Cordero del C. et al. Parasitología Veterinaria, Mc Graw Hill, 1999.

11. Cordovés, C. O. Carrapato controle ou erradicao. $2^{\mathrm{a}}$ ed. Livraria Editora Agropecuária, Guaiba Rs-Brasil. 1997.

12. Couto G., N. R. Medicina interna pequeños animales. $2^{a}$ ed. Editorial Intermédica, Buenos Aires, Argentina, 2000.

13. Cholich L. A., Moriena R. A., Álvarez J. D. Identificación de hemoprotozoarios causante de la babesiosis canina en la ciudad de Corrientes. En: Comunicaciones Científicas y Tecnológicas, São Paulo, Brasil. 2004.

14. Greene R. T. Canine ehrlichiosis: Clinical implications for humoral factors. En: Bonagura J. D.: Kirk's Current Veterinary Therapy, Small Animal Practice XII. W.S. Saunders, Philadelphia, U.S.A. 1995.

15. Guglielmone AA, Bechara, GH, Szabó, MP, Barros, DM, Faccini JL, Labruna $\mathrm{MB}$, et al. Garrapatas de importancia médica y veterinaria: América Latina y el Caribe. International Consortium on Ticks and Tick-borne Diseases (ICTTD-2). The Netherlands: p. 4. 2004.

16. Hendrix, Ch. M., Diagnóstico Parasitológico Veterinario, $2^{\underline{a}}$ Ed. Harcourt Brack. México, 1999.

17. Hoskins, J. D. Ehrlichial diseases of dogs: diagnosis and treatment, Can. Pract. 16: 13-21. 1991.

18. López, V., G. Control de garrapatas. Instituto Colombiano Agropecuario, ICA Regional 4, Medellín. Compendio N. 39. 1980.

19. López, J. Ehrlichiosis canina. MEVEPA 13 (2): 34-35. 1999. 
20. Ristic M., Holland C. Ehrlichiosis canina. En: Manual de las enfermedades infecciosas en pequeños animales. $1^{\underline{a}}$ ed., Editorial Médica Panamericana, S.A. Buenos Aires, Argentina. 1992.

21. Magela M. S., Machado R., Lygia Friche Passos, Detection of Ehrlichia canis in bone marrow aspirates of experimentally infected dogs, 35 (4). 2005.

22. de Tamí I del C., Tamí M. I. M. Identification morphological of Ehrlichia sp. In the plaquets of patients En: Pan American Journal of Public Health, Venezuela. 16 (5): 345-349. 2004

23. Wood B. J., Hoskins J. D. Ehrlichial diseases of dogs. Vet Clin. N. Am., 21: 7598. 1991. 